\title{
Dimensiones del profesionalismo médico. Proyecciones para el siglo XXI
}

\author{
Eduardo Rosselot J.
}

Dimensions of medical professionalism. Perspectives for the twenty-first century

Medicine is progressively loosing its prestige and efficacy and needs to rebuild the moral values that maintain the tacit social contract subscribed between doctors and patients. This altruistic commitment to serve people has been devaluated by changes in health systems, by advances in knowledge and by social and economical changes. The perspective with which humanity faces its future, harmed by radical environmental transformations also has contributed to this devaluation. Notwithstanding, requirements to provide high quality health care and demands to reinstall those fundamental attributes of professional work, commit us to introduce or increase the selective learning of those competences to health professionals. This new, or renewed form of improving the teaching of health sciences, seems to be the unique way to stop the reduction on health care professional effectiveness, to maintain quality of care and to accomplish the goal of medical profession to serve and take care of humankind health (Rev Méd Chile 2006; 134: 657-664).

(Key words: Ethics; Ethics, medical; Practice management, medical; Profesionalism; Sociology)

Recibido el 25 de julio, 2005. Aceptado el 23 de agosto, 2005.

Trabajo basado en la ponencia homónima presentada en el panel sobre Profesionalismo llevado a cabo en el XXVI Congreso de Medicina Interna en Viña del Mar, el 29 de octubre de 2004.

Departamento de Educación en Ciencias de la Salud y Departamento de Bioética y Humanidades Médicas, Facultad de Medicina, Universidad de Chile.

$\mathrm{S}^{\mathrm{e}}$ er un profesional es más que dominar una écnica. Se inscribe en nuestra naturaleza moral: es materia no sólo de mente y de brazos, sino de corazón; no sólo de intelecto y competencias sino que de carácter. Porque sólo habiéndose dedicado y estando apto para comprometerse con otros y servirlo con alta capacidad, es que una persona hace pública profesión de su camino en la vida. (Kass LR.) ${ }^{1}$.

La inquietud por hacer consistentes los valores centrales de la profesión médica, cuando se asiste a su alteración o supresión, ha llevado a diferentes organismos societarios internacionales, y también en nuestra región, a reformular en términos contemporáneos, los conceptos que orientan las decisiones en medicina ${ }^{2-6}$. Concordantemente, ha resurgido una razonable preocupación por perfeccionar los contenidos del aprendizaje en las profesiones de la salud, con los valores éticos que, en el marco de las relaciones entre profesionales y de proveedores-usuarios de los sistemas de salud, caracterizan más apropiadamente la excelencia del ejercicio profesional. 
Actualizar tales valores requiere tomar en cuenta los factores que han restringido su vigencia y considerar, especialmente, su aplicabilidad a las condiciones en que se ejerce la medicina, y que adulteran su sentido, desconocen su significación o reducen su importancia. Resalta la necesidad de recuperar el interés de quienes se forman profesionalmente, por vivir esos valores, expresión de una efectiva calidad de la profesión pese a que la cultura ambiente se excusa de hacerlos propios, en un afán de modernidad y deshumanización que no deja de sorprender ${ }^{7-9}$.

Es indispensable encontrar sentido e implicancia a las nuevas formulaciones valóricas que se sugiere incorporar, porque son más atinentes a los actuales escenarios de la relación clínica, en el modelo de salud vigente, donde cada protagonista en la relación médico paciente, adquiere distinta expresión.

\section{RELEVANCIA DEL PROFESIONALISMO EN MEdicina}

No debemos olvidar que los fines de la medicina han sido remodelados en razón de los cambios que el mundo ha ido experimentando, en las últimas décadas, en el contexto de la salud, el desarrollo científico tecnológico, los factores socio-políticos y culturales que conmocionan sus acciones, propósitos y alcances ${ }^{10-12}$. Lejos de insistir en liberar al hombre de la enfermedad y la muerte, como pudiera haber sido la expectativa de la medicina actual, la visión sociológica- humanista con que se revisten las profesiones de la salud, privilegiando lo colectivo y la equidad para sanar la vida, las hace converger a la prevención y promoción, al alivio y el evitar el mayor daño, toda vez que ha sido planteada ya, $\mathrm{y}$ aceptada, la natural realidad de la muerte ${ }^{13}$.

Esta nueva actitud ante la enfermedad y la muerte no es ajena al progresivo y masivo envejecimiento de la población que surge de los avances en el control de la enfermedad, el alargue de las expectativas de vida, los compromisos que impone esto al resto de la sociedad y las consideraciones suscitadas, respecto a la calidad de vida y los recursos que demanda su sustentación (Tabla 1). Factor común a los elementos que inciden y resultan del mejor control de la salud, es el incremento de costos al requerirse mayor prevención, promoción, perfeccionamiento de la pesquisa, refinamiento en los diagnósticos, incorporación de más tecnología, capacitación e investigación asociadas. Así, se asegura equidad para una medicina asentada como un derecho de la humanidad, lo que a la vez entraña inversiones que difícilmente pueden cubrirse en regímenes de gobierno y economías regidas por premisas de libre mercado. Sobre todo, si más que como un derecho de la humanidad se entiende la medicina o la salud como un bien de consumo o como producto negociable de una tecnocracia ${ }^{14}$.

Surge una discrepancia manifiesta entre expectativas y realidades que no queda relegada sólo al nivel de la satisfacción de las necesidades de atención de salud sino que puede ser causa de

Tabla 1. D eterminantes y sentido del requerimiento del profesionalismo

\begin{tabular}{|ll|}
\hline Condición & Consecuencia \\
\hline Envejecimiento de la población & Actitud ante la enfermedad \\
Alza de costos & Equidad \\
Evidencia de la incerteza & Expectativas y realidades \\
Información y gestión & Búsqueda de la eficacia \\
Cambios valóricos & Vocación o retribución social \\
Práctica personal o institucional & Sensibilidad y medición \\
Métodos extraordinarios y ordinarios & Proporcionalidad y efectividad \\
Autonomía y competencia & Decisiones informadas \\
Sociedad del conocimiento y comunicación & Aprendizaje autónomo y renovado \\
\hline
\end{tabular}


honda frustración profesional ${ }^{13}$. De la medicina, en cuanto conocimiento práctico, se derivan conductas y decisiones de trascendentales consecuencias para los individuos y para la sociedad, pero con grados de incertidumbre que serían inaceptables si no estuvieran respaldados por el prestigio profesional al que la sociedad otorga un grado de confianza, tal vez desproporcionado, aunque avalado en su tradicional seriedad ${ }^{15,16}$. También porque, posiblemente, con la información disponible y los atributos desplegados, la búsqueda y la expresión de eficacia han sido una tónica siempre relevante en ella.

A través de la evolución histórica, la profesión médica ha expuesto su preocupación de servicio al prójimo, en variadas formas, en consonancia con los conceptos valóricos predominantes en diversas culturas o ideologías. Sea por vocación o en cumplimiento de un contrato social, un anhelo de proyección externa ha caracterizado su expresión pública y su sentido de solidaridad. Ambas características del trabajo médico revelan atributos personales volcados hacia los destinatarios pero, expresión, al fin, de la propia voluntad del profesional. Los nuevos sistemas o modelos de atención invocan otro dinamismo, en que el atractivo está en el sistema y el profesional es sólo el demandado a prestar su acción, en una praxis personal o como parte de una institución. Su ejercicio es medido y apreciado en cuanto a sensibilidad, capacidad de respuesta y efectividad del acto. En este orden, tiene sentido considerar la proporcionalidad de las acciones respecto, esencialmente, al riesgo involucrado en la enfermedad; también, en relación al costo, consecuencias o efectividad de las intervenciones médicas, de donde suele desprenderse lo ordinario o extraordinario de los procedimientos involucrados.

En la magnitud y consecuencias de los efectos de las intervenciones médicas se puede deducir la eficacia y la pertinencia de los procesos o las acciones de salud. En esto, la medicina actual destaca la importancia de la autonomía y la determinación del grado de competencia o capacidad del beneficiario de la acción de salud, para decidir autónomamente sobre su compromiso con las funciones curativas individualizadas. Tal autonomía está supeditada a la información recibida, en virtud de que ella ilustra y capacita para tomar decisiones con plena libertad.
No puede ser de otra manera en la sociedad del conocimiento y comunicación que constituimos y por cuyas características se ha condicionado específicamente tanto la sensibilidad $\mathrm{y}$ respuesta de los usuarios como los atributos y formación de los proveedores, orientados por un aprendizaje autónomo y renovado.

Esta concatenación de circunstancias, configura un perfil, exigible a los profesionales, cuyos rasgos pueden resumirse en diversas competencias vinculadas a los principios y compromisos que se han definido como propios del profesionalismo. Tanto éstos como las condiciones en que se desarrolla la medicina y sus consecuencias, sucintamente analizadas, forman un reticulado de interacciones que es comprensible porque las características del perfil traducen los compromisos que determina la profesión y son éstos, a la vez, los que establecen las competencias que deben configurar los atributos y aptitudes propias de su ejercicio (Tabla 2).

Este entramado, de alguna manera, revela la necesidad de utilizar integradamente las oportunidades de aprendizaje que se desarrollan en la educación formal del pre y postgrado ${ }^{17,18}$. De ahí procede ir reforzando aspectos valóricos de los alumnos, en estos atributos morales de los cuales no puede prescindir ni la profesión, como un colectivo humano que posee, desarrolla y perfecciona sus capacidades para el servicio de la sociedad, ni sus integrantes individuales ${ }^{19}$. De ello depende la calidad y pertinencia de su ejercicio y la trascendencia que, en el ámbito de la actividad, manifiesta la idoneidad de sus constituyentes. La preocupación por la formación valórica, que se advierte renacer en la mayor parte de las Escuelas de Medicina o de las profesiones de la salud, es expresión de este imperativo al que convocan las instancias docentes, académicas $\mathrm{y}$, también, las responsables de la provisión de servicios, en todos los ámbitos donde se ejercen acciones formales para la salud humana ${ }^{20,21}$. Igualmente, forma parte de los contenidos de la formación continua y es exigible en los procesos de aseguramiento de la calidad como estándares, no exclusivamente cognitivos, que deben ser mantenidos, respetados y exigidos para dar fe de la propiedad de la formación y de su eficacia ${ }^{22,23}$.

Persiste el desafío por definir acabadamente y poder medir los atributos no cognitivos, las 
Tabla 2. Principios, compromisos y competencias del profesionalismo

\begin{tabular}{|lll|}
\hline Principios y compromisos* & I & Competencias** \\
& $\mathbf{N}$ & \\
Primacía del bienestar del paciente & $\mathbf{T}$ & Habilidad para hacerse cargo de la salud \\
Relevancia de la autonomía & $\mathbf{R}$ & de la comunidad \\
Justicia social & $\mathbf{A}$ & Proveer cuidados de salud contingentes \\
Competencia profesional & $\mathbf{C}$ & Asegurar cuidados apropiados y costo \\
Honestidad & $\mathbf{C}$ & efectivos \\
Confidencialidad & $\mathbf{I}$ & Promover estilos de vida saludables \\
Relaciones apropiadas & $\mathbf{N}$ & Proporcionar consejos en materias \\
Perfeccionar la calidad de la atención & $\mathbf{E}$ & bioéticas \\
Mejorar el acceso a la atención & $\mathbf{V}$ & Estar capacitados para el aprendizaje \\
Distribución justa de recursos limitados & $\mathbf{A}$ & permanente \\
Desarrollo del conocimiento científico & $\mathbf{R}$ & Actuar en un sistema pluralista, con \\
Mantener confianza manejando los & $\mathbf{I}$ & diversidad racial y cultural \\
conflictos de intereses & $\mathbf{D}$ & Ejercer con profesionalismo \\
Responsabilidad profesional & $\mathbf{A}$ & \\
\hline
\end{tabular}

***Principios, compromisos y competencias (así como los condicionantes y consecuencias [Tabla 1]) interaccionan para influirse mutuamente y configurar los atributos y aptitudes del perfil profesional.

actitudes y las destrezas características de la conducta profesional, tanto en los estudiantes como en quienes ya ejercen en este campo. Como ello constituye una preocupación actual relevante de las instituciones formadoras, lo mismo que de las corporaciones que necesitan emplear servicios de salud de calidad y de los pacientes a quienes tales servicios van a ser ofrecidos, es posible encontrar diversas propuestas en evaluación, destinadas a perfilar precondiciones y hábitos de conductas logrados. Son dignos de evaluar, como parámetros valóricos perceptibles en cohortes estudiantiles, los que proponen Mann y cols, en un listado objetivo, donde han apreciado en estudiantes canadienses, con distintos grados de sensibilidad de los rasgos investigados, la probable utilidad para identificar valores profesionales (Tabla 3) y de medir también sus fallas en un marco referencial de actitudes ${ }^{24}$. Éstas o similares descripciones de comportamientos, constituyen los parámetros donde se pueden identificar valores como elementos del profesionalismo o de su carencia. Su incorporación, en calidad de competencias no cognitivas, atributos o destrezas, a los contenidos del currículum y a los procedimientos de evaluación, permiten disponer condiciones de aprendizaje necesarias $\mathrm{y}$, en definitiva, mensurables $^{29-33}$.

Es posible distinguir, en el siglo reciente, dos o tres hitos incidentes en la educación en ciencias de la salud que es necesario advertir para entender mejor las transformaciones acaecidas en la educación superior y sus actuales orientaciones ${ }^{34}$. Aunque puede haber tenido significado, inicialmente, sólo para el ámbito local, el análisis de Flexner sobre la educación médica norteamericana en 1910, no tardó en repercutir mundialmente sobre la organización académica y la educación en salud, de manera radical. Un segundo elemento determinante podemos referirlo a la declaración de la OMS en Alma Ata, en 1978, donde -haya sido o no definitiva- una nueva concepción de la salud estableció condiciones de demanda y provisión que significan un cambio cultural y sociológico que arrastra necesariamente la formación profesional a sus premisas. Por último, el fenómeno de globalización que estamos advirtiendo y viviendo, y que a través de promover y provocar cambios trascendentales en la organización social, educativa, económica y política, aun cuando ha dejado hasta ahora «ntocada»la formación médica ${ }^{35}$, en sus grandes líneas desafía al 
Tabla 3. Conductas analizables en estudiantes y profesionales de la salud relacionadas con profesionalismo*

1. Actúan con honestidad e integridad.

2. Revelan compasión y empatía en el cuidado de pacientes.

3. Actúan de manera confiable y veraz.

4. Interactúan efectivamente con pacientes y sus familias.

5. Muestran compromiso por aprendizaje continuo.

6. Muestran respeto por los pacientes.

7. Crean confianza con los pacientes.

8. Desarrollan sensibilidad a las necesidades de los pacientes.

9. Trabajan como integrantes de un equipo de salud.

10. Demuestran responsabilidad y confiabilidad.

11. Interactúan efectivamente con otros profesionales de la salud.

12. Respetan la privacidad, confidencialidad y dignidad de los pacientes.

13. Reconocen sus limitaciones en destrezas y conocimientos.

14. Muestran buenas relaciones con colegas.

15. Desarrollan sentido del deber.

16. Cuidan eficaz, digna y compasivamente a pacientes moribundos.

17. Entienden y respetan los roles de otros profesionales de la salud.

18. Reconocen los dilemas éticos que ocurren en el aprendizaje y la práctica.

19. Privilegian el interés del paciente sobre el interés personal.

20. Perciben los dilemas éticos mayores en medicina.

21. Se muestran sensibles a factores culturales.

22. Entienden la naturaleza del contrato social de la profesión.

23. Demuestran visiones no juzgadoras de otros valores.

24. Comprenden sus propios valores.

25. Equiparan demandas de la vida personal y profesional.

*Modificado de Mann et al ${ }^{24}$.

El estudio fue realizado en 4 grupos (alumnos, residentes, docentes y profesionales de la salud no médicos); los resultados difieren entre ellos, pero en general, las 5 primeras conductas guardan la mejor correlación con valores profesionales y parece claro (puede ser no evidente) que son aprendibles, si no han sido integrados al comportamiento personal antes de ingresar a los estudios profesionales. Es cierto también que puede producirse detrimento de ellos durante el aprendizaje, especialmente por efecto del curriculum oculto (papel de los malos modelos y sus incoherencias) y la desprofesionalización (erosión ética) en el ejercicio de la medicina ${ }^{25-28}$.

sistema educacional en ciencias de la salud que tendrá, al fin, que amoldar sus estructuras y funcionalismo a los requerimientos avasalladores de la universalidad ${ }^{36}$.

\section{EL PROFESIONALISMO EN LA EDUCACIÓN EN LAS CIENCIAS DE LA SALUD}

Sería paradójico que el conjunto de valores exigibles en los alumnos y en los profesionales de las ciencias de la salud como elementos paradigmáticos de la excelencia en su quehacer, no lo fueran así ni estuvieran implícitamente presentes en quienes promueven el aprendizaje de tales atributos o destrezas, en el aula, los laboratorios, los escenarios clínicos. Pero esto representa todo un capítulo de los textos concentrados en gestión educacional y, salvo renombradas circunstancias y ejemplares personajes ${ }^{37}$, los datos y la casuística contemporánea dan cuenta, más bien, de los contrastes y frustraciones de los alumnos respecto 
a quienes debieran ser, para ellos, emulables y motivantes. Por lo menos, se describen, a menudo, como elementos del currículum informal $u$ oculto, y son causa de decepción, sino indignada contemplación, de quienes mantienen una imagen idealizada de compromiso y servicio, de médicos, enfermeras y matronas, que alguna vez hicieron relevante, por su honorabilidad, calidad y altruismo, esas disposiciones ${ }^{25,26,38}$.

Pero, tener aptitudes docentes fue siempre propio de un buen profesional, a quien bastaba proponerse a entregar sus conocimientos -como un virtuoso musical pudiera enseñar el canto con sólo ser imitado al interpretar una melodía- en el lugar donde ejerce sus habilidades. Pese a que el fenómeno prevalente hoy día, de proliferación de escuelas de la salud, sin la necesaria planificación, disponibilidad de recursos ni cometido educacional de experiencia, no ha superado estas deficiencias, ya no es más concebible asumir tareas educativas sin un saber metodológico y procedimental, que respalde la facilitación del aprendizaje que, en sus alumnos, el docente debe inducir y el sistema promover ${ }^{39,40}$.

Por eso es que el profesionalismo, como cúmulo de virtudes, aptitudes no cognocitivas, atributos, competencias generales y destrezas específicas, surge hoy día como un requerimiento esencial de una formación idónea, que se manifiesta exigentemente en el cuerpo académico y debe estar incorporada en todo el proceso de aprendizaje de quienes adquieren las competencias resaltadas como parte de su bagaje profesional ${ }^{41-43}$.

Alcanzar estas dotes supone, por una parte, profundizar en las habilidades y conocimientos basados en evidencia, lo que quiere decir que el docente no puede, tampoco, dejar de desarrollar su propio caudal de información, elaborarlo críticamente, evaluar su consistencia, y aplicarlo en su propio campo para suscitar modificaciones y mejoras de acuerdo a los resultados que se vayan acumulando 44,45. Es así como la investigación educacional forma parte integrante e irrenunciable de este quehacer docente. Aún más, este profesional, dado su compromiso docente, deberá mantener una reflexión permanente referida a sus valores como profesional y como facilitador del aprendizaje y de la formación de sus alumnos; sólo así podrá detectar las acechanzas a la educación y los vicios, tanto formales como virtuales, del currículum. Con mayor razón que a los profesionales que desarrollan apropiadamente su actividad en la atención clínica, cualquiera sea el sistema de salud donde intervenga, las amenazas al profesionalismo se aplican con mayor vigor y persistencia contra los docentes, tanto porque éstos ejercen relevantemente el rol de modelos, como porque las mismas acechanzas y desviaciones del profesionalismo provienen de las distintas vertientes de los sistemas de salud, del medio universitario, de los usuarios de la educación y las agencias o instituciones que conviven o gestionan los servicios de salud ${ }^{46-48}$ (Tabla 4). Ésta es una de las razones de por qué es necesario identificar las áreas que requieren especial dedicación para cautelar la excelencia y calidad en el desarrollo profesional de los estudiantes ${ }^{40-43,49}$. Se ha ido estableciendo un consenso de que -a través de una adecuada selección de los estudiantes y de la vigilancia sobre el currículum informal-, la elección de profesores como buenos modelos, el resguardar la diversidad y los aspectos multiculturales de la práctica médica y el establecer trabajo en equipo en los cuidados de salud primarios y secundarios, constituyen herramientas de gran importancia para conseguir los objetivos de una formación profesional idónea $26-28,48-50$.

\title{
Tabla 4. Amenazas al profesionalismo
}

\author{
El incentivo del lucro en el ejercicio profesional (mercadismo). \\ La postergación del mayor bien del paciente o del alumno por la prevalencia del sistema. \\ Los incentivos espúreos para controlar costos. \\ El predominio de los objetivos organizacionales. \\ El impacto sobre los alumnos de las actitudes y comportamiento de los docentes.
}




\section{REFERENCIAS}

1. KAsS LR. I will give no deadly drug. Bull Am Coll Surg 1992; 77: 6-17.

2. General Medical Council. Tomorrow's Doctors. Recommendations on Undergraduate Medical Education. London. 1993, y Good Medical Practice. London 1993.

3. Colegio Médico de Chile A.G. Decálogo de la buena práctica médica. Santiago. 1999.

4. Rosselot E. Reivindicando la medicina como profesión científico-humanista. Rev Méd Chile 2003; 131: 454-6.

5. REYNOLDS PP. Reaffirming Professionalism through the Education Community. Ann Int Med 1994; 120: 609-14.

6. Medical Professionalism in the New Millenium: A Physician Charter. Project of the ABIM-ASIM Foundation and European Federation of Internal Medicine. Ann Int Med 2002; 136: 243-6. Ibid. (traducción) Rev Méd Chile 2003; 131: 457-60.

7. Thompson JN. Moral Imperatives for Academic Medicine. Acad Med 1997; 72: 1037-42.

8. ReLman AS. Education to Defend Professional Values in the New Corporate Age. Acad Med 1998; 73: 1229-33.

9. Horwitz N. El sentido social del profesionalismo médico. Rev Méd Chile 2006; 134: 528-32.

10. Goic A. El Fin de la Medicina. Mediterráneo, Santiago. 2000.

11. Jonas H. The Imperative of Responsability. In Search of an Ethics for the Technological Age. University of Chicago Press. 1984.

12. Wolf SM. Health Care Reform and the Future of Physician Ethics. Hasting Center Report 1994; 24: 28-41.

13. Sмттн R. Why are doctors so unhappy? BMJ 2001; 322: 1073-4.

14. Lolas F. La praxis médica en la interfase de ética, política y técnica, en Taller: Multiprofesionalidad en el Campo de la Salud. Santiago, noviembre 6, 1966. Universidad de Chile. 11-21.

15. Feinstein AR, HuRwitz RI. Problems in evidence of evidence based medicine. Am J Med 1997; 103: 529-35.

16. INUI TS. A Flag in the Wind. Educating for Professionalism in Medicine. AAMC 2003.

17. Wear D, Castelani B. The Development of Professionalism. Curriculum Matters. Acad Med 2000; 75: 602-11.
18. FranckFord DM, KonRad TR. Responsive Medical Professionalism: Integrating Education, Practice and Community in a Market-driven. Era Acad Med 1998; 73: 138-45.

19. Cruess RL, CRuess SR. Teaching Medicine as a Profession in the Service of Healing. Acad Med 1997; 72: 941-51.

20. Howe A. Professional Development in Undergraduate medical Curricula - the Key to the Door of a New Culture. Med Education 2002; 36: 353-9.

21. SPencer J. Teaching About Professionalism. Med Education 2003; 288-9.

22. PARDELL H. ¿Tiene sentido hablar de profesionalismo hoy? Educación Médica 2003; 6: 63-80.

23. Stern DT, Frohna AZ, Gruppen LD. The prediction of professional behaviour. Med Education 2005; 39: 75-82.

24. Mann KV, Ruedy J, Milar N, Pantelis A. Achievement of non-cognitive goals of undergraduate medical education: perceptions of medical students, residents, faculty and other health professionals. Med Education 2005; 39: 40-8.

25. SteRn D. Practicing what we preach? An Analysis of the Curriculum of Values in Medical Education. Amer J Med 1998; 104: 569-75.

26. KenNy NP, MANN KV, MaCLEOD H. Role modeling in physicians' professional formation: Reconsidering an essential but untapped educational strategy. Academic Medicine 2003; 78: 1203-10.

27. Feudtner C, Christakis DA, Christakis NA. Do clinical clerks suffer ethic erosion? Students perception of their ethical environment and personal development. Acad Med 1994; 69: 670-9.

28. REED RR, Evans D. The Deprofessionalization of Medicine. Causes, Effects and Responses. JAMA 1987; 258: 3279-82.

29. Medical School Objectives Writing Group. Learning objectives for Medical Student Education. Acad Med 1999; 74: 13-8.

30. Neufeld VR, Maudsley RF, Pickering JM et al. Educating future physicians for Ontario. Acad Med 1998; 73: 1133-48.

31. Can Meds 2000 project. Skills for the New Millenium. Report of the Societal Needs Working Group. Ottawa, on: Royal College of Physicians and Surgeons, 1996.

32. EPSTEIN RM, Hundert EM. Defining and Assessing Professional Competence. JAMA 2002; 287: 226-35.

33. Accreditation Council for Graduate Medical Education Outcome Project Advisory Committee. The 
ACGME Outcome Project. Enhacing Residency Education through Outcomes Assessment. Chicago, IL: ACGME 1999. http://www.acgme.org/ outcome/project/proHome.asp (accessed 20 de enero de 2004)

34. Distlehorst LH, Dunnington GL, Roland Folse J. Teaching and learning in medical and surgical education. Lessons learned for the $21^{\text {st }}$ century. Southern Illinois University School of Medicine. Lawrence Erlbaum Ass NJ 2000.

35. Christensen L. The Bologna Process and Medical Education. Med Education 2004; 26: 625-9.

36. Rosselot E. Realidad y Proyecciones de la Educación en Ciencias de la Salud en Chile. Anales de la Universidad de Chile 2004; 16: 71-96.

37. Goic A. Grandes Médicos Humanistas. Ed. Universitaria. 2004.

38. HaFFERTY FW, Frank R. The Hidden Curriculum, Ethics Teaching and the Structure of Medical Education. Acad Med 1994; 69: 861-71.

39. Arnold EL, Blank LL, Race KEH, Cipparrone N. Can Professionalism be measured? The Development of a Scale for Use in the Medical Environment. Acad Med 1998; 73: 1119-21.

40. ArNold L. Assessing Professional Behaviour: Yesterday, Today, and Tomorrow. Acad Med 2002; 77: 502-15.

41. Armstrong EG, Doyle J, Bennett NL. Transformative Professional Development of Physician as
Educator: Assesment of a Model. Acad Med 2003; 78: 702-7.

42. Kuein EJ, Jackson C, Kratz L ET aL. Teaching Professionalism to Residents. Acad Med 2003; 78: 26-34.

43. Phelan S, Obenshain S, Galey WR. Evaluation of the Noncognitive Professional Traits of Medical Students. Acad Med 1993; 68: 799-803.

44. Hatem CJ. Teaching Approaches that Reflect and Promote Professionalism. Acad Med 2003; 78: 709-13.

45. Harden RM, Lumey PM. Best Evidence Medical Education: the single truth. Medical Teacher 2000; 22: 117-9.

46. LUDMERER KM. Instilling Professionalism in Medical Education. JAMA 1999; 282: 881-2.

47. Swick HM, Szenas P, Danoff D, Whitcomb ME. Teaching Professionalism in Undergraduate Medical Education. JAMA 1999; 282: 830-2.

48. BALDWIN DC, DAUGHERTY SR, RowLEY BD. Unethical and Unprofessional Conduct Observed by Residentes during Their First Year of Training. Acad Med 1998; 73: 1195-200.

49. Edwards JC, Elam CL, Wagoner NE. An Admission Model for Medical Schools. Acad Med 2001; 76: 1207-12.

50. Stephenson A, Higgs R, Sugarman J. Teaching Professional Development in Medical Schools. Lancet 2001; 357: 867-70. 